Ann. Zootech., I975, 24 (2), I99-207.

\title{
INFLUENCE DU MODE DE CONDITIONNEMENT DES FOURRAGES DÉSHYDRATÉS SUR LES FERMENTATIONS DANS LE RUMEN DU MOUTON : COMPARAISON ENTRE LÉGUMINEUSES ET GRAMINÉES
}

\author{
C. MASSON, M. CANDAU et J.-L. TISSERAND \\ avec la collaboration technique de C. Cordeler et J. Berthier \\ Laboratoire de Recherches de la Chaire de Zootechnie de l'E. N. S. S. A. A., I. N. R. A., \\ 26, Bd Dr Petitjean, \\ 21016 Dijon Cedex
}

\section{RÉSUMÉ}

Neuf moutons munis de fistule du rumen nous permettent d'étudier l'influence du conditionnement de fourrages déshydratés : trois graminées et deux légumineuses présentées sous trois formes : normale (hachée), comprimée (agglomérée dans une presse à piston), compactée (agglomérée sans broyage préalable dans une presse à filière de $8 \mathrm{~mm}$ ).

La taille moyenne des particules de fourrage varie selon le mode de conditionnement de $0,45 \mathrm{~mm}$ à $4,05 \mathrm{~mm}$ (tabl. $\mathrm{r}$ ).

Le mode de conditionnement influe de façon significative sur le niveau d'ingestion (tabl. I), et peu sur la production d'acides gras volatils (AGV) in vitro (tabl. 2), sauf pour le trèfle violet. Par contre, la composition du mélange des acides gras volatils du rumen est modifiée par le mode de conditionnement et la nature du fourrage. Avec les graminées, et par rapport à la forme hachée, les formes comprimées et compactées entraînent une diminution du pourcentage d'acide acétique (- 4,4 points et $-5,3$ points) compensée par une augmentation d'acide propionique $(+\mathrm{I}, 4$ points et 5,o points). Par contre, avec les légumineuses, les formes comprimées et compactées entraînent une augmentation du pourcentage d'acide acétique $(+7,8$ points et + Io,o points) compensée par une baisse du pourcentage d'acide propionique et d'acide butyrique.

Une mesure de l'activité fermentaire du rumen (tabl, 3) in vivo $(\mathrm{pH}, \mathrm{AGV})$ en fonction du moment de prélèvement confirme les observations faites par divers auteurs.

\section{IN'TRODUCTION}

Le conditionnement des fourrages sous forme broyée et agglomérée (dite condensée) a fait l'objet de nombreux travaux (BLAXTER et GraHAM, I956; MrNSON, I962 ; MoOre, r964; BeARdsley, I964; Journet et DEMARquIliy, I967 ; CotTYN et Boucque, I968; Colin, I968; Thomson et al., I972; JARrige et al., I973). 
Ce mode de présentation réduit le fourrage en fines particules, ce qui permet d'accroître les quantités ingérées par les animaux mais, par contre, entraîne une baisse de la digestibilité de la matière organique, une diminution du temps de rumination, une déviation des fermentations dans le rumen et éventuellement des accidents sanitaires. Pour pallier ces inconvénients, il est envisagé de ne pas broyer le fourrage déshydraté et de le presser directement, soit dans une presse à piston (fourrage comprimé), soit dans une presse à filière (fourrage compacté). Cependant l'influence sur la valeur alimentaire de ces modes de conditionnement des fourrages reste encore assez mal connue. Nous nous sommes donc préoccupés depuis r 969 de mettre en évidence les effets de ces différentes formes de présentation sur la granulométrie, le niveau d'ingestion et les fermentations dans le rumen. L'étude a été effectuée sur trois graminées et deux légumineuses.

\section{MATÉRIEL E'T MÉTHODE}

Schéma expérimental

\section{Fourrages.}

Les légumineuses utilisées sont une luzerne de I er cycle récoltée au stacle début floraison, un trèfle violet de $\mathrm{I}^{\mathrm{er}}$ cycle et de $2^{\mathbf{e}}$ cycle. Les graminées sont représentées par un ray-grass, un dactyle et une fétuque élevée, toutes trois récoltées au stade épiaison du i ${ }^{\text {r }}$ cycle.

\section{Animaux.}

Ces essais sont réalisés sur 9 moutons adultes (Ile de France) munis de fistule clu rumen et répartis en trois lots comparables au point de vue poids et niveau de consommation.

\section{Protocole expérimental.}

Chaque fourrage est distribué successivement aux trois lots de montons sous trois formes de présentation : forme hachée, forme compriméc dans une presse à piston, forme compactée dans une presse munie d'une filière de $8 \mathrm{~mm}$.

Chaque essai dure six semaines et comporte:

- une phase d'accoutumance à chaque régime de 4 semaines,

- une phase expérimentale proprement dite de 2 semaines.

\section{Mesures}

\section{Composition chimique.}

Les teneurs en matière sèche, cendres, azote, cellulose brute (Weencle) de chaque fourrage sont déterminées selon les méthodes officielles de l'A. F. Z. sur un échantillon moyen de chaque fourrage constitué au cours de chacune des périodes.

\section{Granulométrie.}

Les mesures sont faites sur trois échantillons de $50 \mathrm{~g}$ de fourrage. L.es agglomérés sont désagrégés à la main après humectation à l'aide d'une pissette. Après un séchage naturel à l'air, le fourrage est tamisé pendant $5 \mathrm{mn}$ puis Io $\mathrm{mn}$ sur un appareil Vibreur Tamisor (méthode mise au point par DEMARQUILLY).

Les critères retenus sont la taille moyenne des particules et les pourcentages de particules supérieures à $\mathrm{I}, 25 \mathrm{~mm}$ ct $0,80 \mathrm{~mm}$. 


\section{Quantités ingérées.}

Une quantité d'aliment connue est distribuée à chaque mouton en deux repas à $8 \mathrm{~h}$ et $\mathbf{I} 6 \mathrm{~h}$. L'aliment est distribué à volonté mais, pour éviter une trop grande perte, les rations sont ajustées en fonction de la consommation des jours précédents de telle sorte que les refus se situent entre Io et 20 p. Ioo de la quantité distribuée.

Les refus sont enlevés et pesés chaque jour avant la distribution du repas du matin.

\section{Analyses}

\section{Digestion in vivo.}

Dans le cas de la Luzerne et sur deux moutons par type de régime, nous avons suivi l'évolution de l'activité fermentaire du jus de rumen au cours de la journée. Les critères retenus sont le pH, la concentration et la composition en acides gras volatils.

Le fourrage et l'eau sont retirés aux animaux après le repas du soir précédant le jour de la mesure. Le lendemain, des prélèvements de jus de rumen sont effectués à partir du temps o, c'esta-dire avant le repas du matin, puis $I h, 2 h, 3 h, 5 h, 6 h$, après le repas qui dure $I h$. Les quantités ingérées sont mesurées avec exactitude. A chaque prélèvement, le jus de rumen est filtré sur six couches de gaze. Le pH est mesuré immédiatement et les échantillons sont congelés pour doser les acides gras volatils ultérieurement.

\section{Digestion in vitro.}

Les études de digestion in vitro sont réalisées à l'aide de la technique de "rumen artificiel " mise au point par TISSERAND et ZELTER (I965). L'activité cellulolytique du jus de rumen est exprimée en pourcentage de dégradation de la cellulose du substrat standard déterminée par la méthode de Weende. La teneur et la composition en acides gras volatils (AGV) du jus de rumen sont mesurées par chromatographie en phase gazeuse (support de chromosorb W. 80-roo mesh imprégné de 25 p. Ioo de N-penty-glycol adipate (NPGA) et 2 p. Ioo d'acide phosphorique).

\section{RÉSULTATS}

Les caractéristiques chimiques ne diffèrent pas selon le mode de conditionnement, comprimé ou compacté, graminées ou légumineuses (tabl. I). Toutefois, il existe quelques variations difficilement explicables en dehors d'un effet d'échantillonnage au niveat de la teneur azotée de la forme comprimée du ray-grass et de la fétuque.

L'analyse granulométrique révèle que le fourrage déshydraté est réduit en particules plus ou moins fines selon le type de presse. La taille moyenne des particules est la plus grande pour la forme hachée et la plus faible pour la forme compactée. Ellle varie de $4,05 \mathrm{~mm}$ pour le dactyle haché à $0,45 \mathrm{~mm}$ pour le trèfle violet compacté $2^{e}$ cycle (tabl. I).

Pour un même mode de conditionnement, les graminées semblent moins sensibles à l'effet du malaxage des presses que les légumineuses.

\section{Quantités ingérées}

A l'exception des trèfles violets, aucune différence significative ne peut être mise en évidence entre les fourrages présentés sous forme hachée et comprimée. Ce phénomène est étroitement lié à la granulométrie des fourrages; en effet, lorsque les fourrages comprimés renferment un pourcentage de particules ( $>1,25 \mathrm{~mm}$ ) supérieur à $50 \mathrm{p}$. Ioo, il semble qu'ils ne soient pas mieux consommés que les fourrages hachés correspondants. 
Le fourrage compacté est, par contre, mieux consommé (plus de $30 \mathrm{p}$. Ioo en moyenne).

Quel que soit le mode de conditionnement, la luzerne est toujours mieux consommée que les autres fourrages et, parmi les graminées, la fétuque est plus appréciée que le dactyle et le ray-grass.

\section{Digestion dans le rumen (tab1. 2)}

a) In vitro.

\section{Activité cellulolytique.}

L'activité fermentaire des contenus de rumen varie peu en fonction du mode de conditionnement. L'activité cellulolytique est moins importante avec les légumineuses qu'avec les graminées; il est possible que ces dernières aient un pouvoir tampon plus faible et une plus grande teneur en glucides solubles fermentescibles.

TABLEAU 2

Mesures in vitro de la digestion dans lo vumen

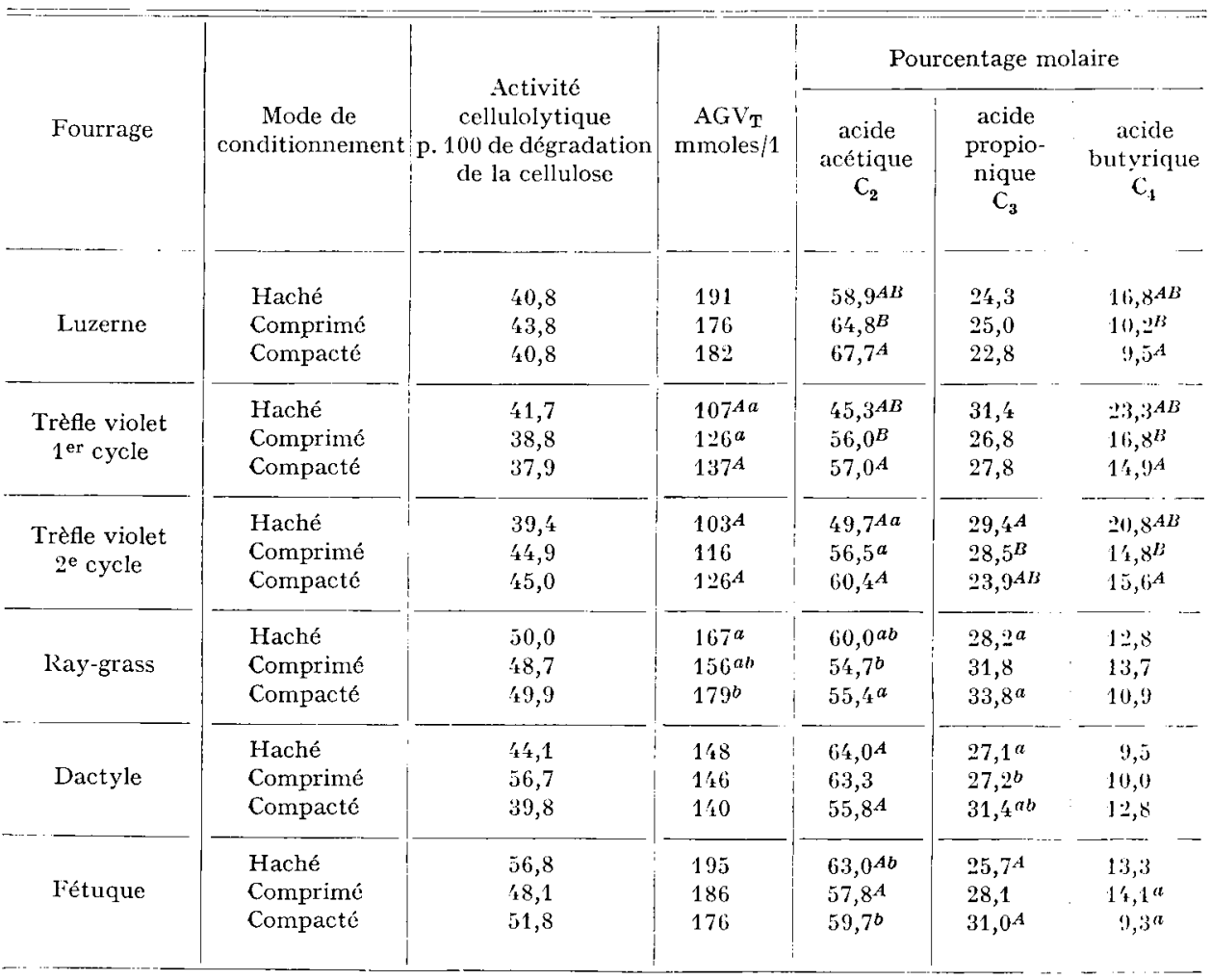

Les valeurs qui portent la même lettre sont significativement différentes :

$$
\begin{array}{ll}
\text { Lettre majuscule } & \mathrm{P}<0,01 \\
\text { Lettre minuscule } & \mathrm{P}<0,05
\end{array}
$$


Production d'acides gras volatils.

La concentration du mélange des $A G V$ du rumen n'est pas modifiée par le mode de présentation, sauf pour le trèfle violet, entre la forme hachée et la forme compactée.

Par contre, la proportion des différents acides gras volatils formés (acides acétique, propionique, butyrique) varie avec la forme de présentation et l'espèce fourragère. En effet, pour les légumineuses, les formes comprimées et compactées entraînent, par rapport à la forme hachée, une augmentation de l'acide acétique $(+7,8$ points et + Io,o points) compensée par une baisse du pourcentage d'acide propionique et notamment d'acide butyrique.

A l'inverse, pour les graminées, les effets des formes comprimées et compactées se traduisent par une diminution du pourcentage d'acide acétique $(-4,4$ points et $-5,3$ points) respectivement compensée par une augmentation d'acide propionique $(+\mathrm{I}, 4$ points et 5,0 points).

\section{TABLEAU 3}

Produits de fermentation dans le rumen à partir de trois modes de conditionnement de fourrage en fonction au moment d'échantillonnage

\begin{tabular}{|c|c|c|c|c|c|c|c|c|}
\hline \multirow{2}{*}{$\begin{array}{l}\text { Moment de } \\
\text { l'échantil- } \\
\text { lonnage }\end{array}$} & \multicolumn{6}{|c|}{ Acides gras volatils (p. 100 molaire) } & \multirow{2}{*}{$\begin{array}{c}\text { Acides } \\
\text { gras totaux } \\
\text { mmole } / 1\end{array}$} & \multirow[b]{2}{*}{$\mathrm{pH}$} \\
\hline & $\begin{array}{c}\text { acide } \\
\text { acétique }\end{array}$ & $\begin{array}{c}\text { acide } \\
\text { propio- } \\
\text { nique }\end{array}$ & $\begin{array}{l}\text { acide } \\
\text { isobuty- } \\
\text { rique }\end{array}$ & $\begin{array}{c}\text { acide } \\
\text { butyrique }\end{array}$ & $\begin{array}{c}\text { acide } \\
\text { isovaléria- } \\
\text { nique }\end{array}$ & $\begin{array}{c}\text { acide } \\
\text { valéria- } \\
\text { nique }\end{array}$ & & \\
\hline \multicolumn{9}{|c|}{ 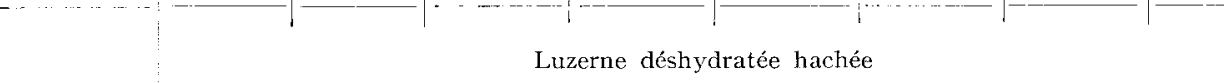 } \\
\hline To & 67,8 & 17,9 & 1,76 & 7,97 & 2,6 & 0,9 & 68,5 & 7,30 \\
\hline $\mathrm{T} 2 \mathrm{~h}$ & 66,1 & 21,0 & 1,61 & 6,9 & 1,5 & 2,2 & 78,6 & 7,14 \\
\hline $\mathrm{T} 3 \mathrm{~h}$ & 67,6 & 20,3 & 1,39 & 6,9 & 1,3 & 2,0 & 76,0 & 7,08 \\
\hline $\mathrm{T} \& \mathrm{~h}$ & 67,0 & 20,2 & 1,33 & 7,6 & 1,6 & 2,1 & 77,8 & 6,99 \\
\hline $\mathrm{T} 5 \mathrm{~h}$ & 69,2 & 18,6 & 1,40 & 7,5 & 1,7 & 1,4 & 77,2 & $7,1^{\prime} t$ \\
\hline \multirow[t]{2}{*}{$\mathrm{T} 6 \mathrm{~h}$} & 69,1 & 18,5 & 1,19 & 7,9 & 1,7 & 1,2 & 73,4 & 7,17 \\
\hline & \multicolumn{8}{|c|}{ Luzerne déshydratée comprimée } \\
\hline To & 68,5 & 17,7 & 1,59 & 8,3 & 2,2 & 1,3 & 68,5 & 7,23 \\
\hline $\mathrm{T} 2$ & 67,5 & 20,2 & 1,07 & 7,6 & 1,3 & 2,3 & 89,9 & 7,11 \\
\hline T 3 & 69,2 & 19,2 & 0,98 & 7,5 & 0,9 & 2,0 & 85,4 & $7,0 \mathrm{~g}$ \\
\hline $\mathrm{T}:_{\mathrm{t}}$ & 67,7 & 20,1 & 0,98 & 7,9 & 0,9 & 2,1 & 80,7 & 7,02 \\
\hline $\mathrm{T} 5$ & 70,9 & 17,8 & 1,00 & 7,7 & 0,8 & 1,6 & 87,6 & 7,11 \\
\hline \multirow[t]{2}{*}{$\mathrm{T} 6$} & 70,0 & 18,0 & 1,11 & 8,4 & 1,1 & 1,1 & 77,4 & 7,16 \\
\hline & \multicolumn{8}{|c|}{ Luzerne déshydratée compactée } \\
\hline$T 1$ & 68,0 & 17,7 & 1,65 & 9,0 & 2,1 & $1,{ }^{\prime}$ & 69,0 & 6,97 \\
\hline $\mathrm{T}:$ & 67,1 & 20,0 & 1,28 & 7,7 & 1,5 & 2,2 & $87, t_{k}$ & 6,81 \\
\hline T 3 & 67,8 & 18,9 & 0,90 & 9,3 & 0,7 & 2,1 & 90,3 & 6,78 \\
\hline $\mathrm{T}^{\prime}$ & 69,1 & 18,9 & 0,91 & 8,0 & 0,8 & 1,9 & 89,3 & 6,81 \\
\hline $\mathrm{T} 5$ & 70,7 & 18,3 & 0,90 & 7,9 & 0,7 & 1,4 & 90,5 & 6,90 \\
\hline $\mathrm{T} 6$ & 71,1 & 17,6 & 1,11 & 7,9 & 1,1 & 1,0 & 74,5 & 7,00 \\
\hline
\end{tabular}


b) In vivo.

Les résultats des mesures in vivo sur luzerne (tabl. 3) confirment les observations faites par plusieurs auteurs (FENNER et al., I967; CoTTYN et BoucQue, I968).

- Le mode de conditionnement a une influence sur la valeur du $\mathrm{pH}$ du milieu ruminal. Le $\mathrm{pH}$ avec le fourrage compacté est significativement plus bas que celui observé avec le fourrage comprimé et le fourrage haché mais il n'y a pas de différence entre le fourrage haché et le fourrage comprimé.

- Le $\mathrm{pH}$ présente une valeur minimum $4 \mathrm{~h}$ après le repas pour les fourrages haché et comprimé et $3 \mathrm{~h}$ après le repas pour le fourrage compacté.

- Les acides gras volatils totaux présentent une valeur maximum entre les temps $T_{2}$ et $T_{5}$. Ces valeurs sont significativement $(\mathrm{P}<0,05)$ plus élevées avec les fourrages compactés qu'avec les fourrages comprimé et haché. tion du temps.

- Les proportions molaires des différents acides gras volatils varient en fonc-

La proportion d'acide acétique diminue après le repas pour remonter aux temps $T_{5}$ et $T_{6}$; par contre, la proportion d'acide propionique augmente au temps ' $T_{2}$ pour diminuer par la suite.

\section{DISCUSSION ETT CONCI,USION}

Les résultats de l'analyse granulométrique des fourrages et des quantités ingérées confirment les observations faites par DEMARQUILLY, JOURNET et JARRIGE.

L'agglomération du fourrage dans une presse à piston respecte mieux la structure physique du fourrage que le passage dans une presse à filière (MELCION et DELORT LAVAL, I972).

Cependant les quantités ingérées de fourrage comprimé sont semblables à celles du fourrage haché correspondant tant que le pourcentage de particules $>1,25 \mathrm{~mm}$ reste supérieur à $5^{\circ} \mathrm{p}$. IOO. Aussi, pour le trèfle violet, l'augmentation significative du niveau d'ingestion entre la forme comprimée et la forme hachée peut être due à un pourcentage de particules > I,25 $\mathrm{mm}$ plus faible de l'ordre de $30 \mathrm{p}$. Ioo.

Toutefois, le fourrage haché de trèfle violet est mal consommé (4I et $5^{\circ} \mathrm{g} / \mathrm{kg}$ $\mathrm{P}^{\mathbf{0}, 75}$ respectivement pour le trèfle violet $\mathrm{I}^{\mathrm{er}}$ cycle et $2^{\mathrm{e}}$ cycle), ce qui peut entraîner une meilleure efficacité du conditionnement.

D'autre part, la baisse du niveau d'ingestion de trèfle violet compacté par rapport au comprimé peut être attribuée à une plus grande dureté des bouchons.

Le mode de conditionnement des fourrages n'entraîne pas de variations significatives de l'activité cellulolytique du jus de rumen. Ces résultats diffèrent de ceux observés par JoURNE'T et HODEN (I973) qui montrent une liaison étroite entre l'activité cellulolytique et la taille moyenne des particules : plus la taille moyenne des particules est petite, plus l'activité cellulolytique diminue.

I, méthode de mesure "des sachets de nylon " utilisée par ces auteurs est susceptible de donner des résultats différents.

La concentration d'acides gras volatils totaux est peu modifiée par le conditionnement des fourrages déshydratés. 
Nos observations sont en accord avec des résultats récents de JoURnet et Hoden (I973), Thouson et al. (I972) qui montrent qu'à niveau d'ingestion constant l'agglomération des fourrages n'entraîne pas d'augmentation significative des $A G V_{T}$ comme le signalent certains auteurs (MINSON, I962 ; BEARDSLEY, I964; COTTYN et BOUCQUE, I968).

Cependant la composition du mélange d'AGV, in vitro surtout, est modifiée sous l'influence du conditionnement, contrairement aux observations de D. J. THomSON et al. (1972), mais conformément aux résultats de WRIGHT et al (I963), CoTTYN et Boucgue (I968). En accord avec ces derniers, nous constatons pour les graminées une baisse de la proportion d'acide acétique et d'acide butyrique et une augmentation de celle d'acide propionique. Mais, pour les légumineuses, nos résultats sont tout à fait différents. Le conditionnement entraîne une augmentation du pourcentage d'acide acétique et une diminution de celui d'acide propionique et surtout d'acide butyrique. Seuls, WESTON et HoGAN (1967) avaient noté ces variations en comparant de la luzerne hachée à de la farine de luzerne. Toutefois il faut signaler que les pourcentages d'acide acétique observés avec des trèfles violets hachés sont particulièrement faibles et que, par conséquent, l'augmentation dûe au conditionnement n'est peut-être pas aussi importante.

D'autre part, le pouvoir tampon des espèces fourragères est variable et, en particulier, celui des légumineuses est plus important que celui des graminées. Ainsi, avec les légumineuses, le pH du jus de rumen étant plus élevé, l'activité des bactéries cellulolytiques serait plus importante, ce qui se traduirait par une augmentation de l'acide acétique et inversement avec les graminées.

Une autre hypothèse pourrait également être avancée : les légumineuses sont, en général, moins digestibles que les graminées par suite de leur degré de lignification plus important. Le conditionnement des fourrages, qui entraîne un certain broyage, pourrait alors, à un instant donné, rendre la cellulose plus accessible aux microorganismes et, par suite, faciliter la formation d'acide acétique. Cependant, avant de retenir telle ou telle hypothèse, il est nécessaire de recourir à une expérimentation.

Reçu pour publication en décembre 1974 .

\title{
SUMMMARY
}

\author{
INFLUENCE OF THE PHYSICAL, FORM OF DEHYDRATED FORAGES \\ ON SHEEP RUMEN FERMENTATIONS : \\ COMPARISON BETWEEN LEGUMES AND GRASSES
}

\footnotetext{
Nine sheep fitted with rumen fistulac were used to study the effect of the physical form of dehydrated forages, three grasses and two legumes, on the fermentations in the rumen. The forages were either chopped (normal form), wafered (agglomerated in a wafering press) or cobbed (agglomerated without previous grinding in an $8 \mathrm{mn}$ die pellet mill).

The mean size of the forage particles varied from $0.45 \mathrm{~mm}$ to $4.05 \mathrm{~mm}$ according to the treatment applied (table I).

The physical form of the forages significantly affected the feed intake level (table I) and only slightly affected the in vitro production of volatile fatty acids (VFA) (table 2), except in the case of red clover. On the other hand, the composition of the mixture of volatile fatty acids in the rumen was modified by the physical form and nature of the forage. With respect to the grasses, the wafered and cobbed forms, as compared to the chopped one, led to a decrease in the percentage of
} 
acetic acid ( -4.4 points and -5.3 points) compensated by an increase in the percentage of propionic acid $(+\mathbf{I} .4$ point and 5.0 points). The contrary was noticed in the case of the legumes in which the wafered and cobbed forms led to an increase in the percentage of acetic acid ( +7.8 points and + ro.o points) compensated by a decrease in the percentage of propionic and butyric acids.

In vivo measurement of the fermentative activity in the rumen (pH, VFA) according to the moment of sampling confirms the observations made by various authors.

\section{RÉFÉRENCES BIBLIOGRAPHIQUES}

BEARDSLEY D. W., I968. Symposium of forage utilisation : nutritive value of forage as affected by physical form. J. Anim. Sci., 23, 239-245.

Blaxter K. L., Graham N. HcC., I956. The effect of grinding and cubing process on the utilisation of the energy and dried grass. J. Agric. Sci., 47, 207.

Colin P., r968. Utilisation par les ruminants d'aliments agglomérés. Doc. S. E. I., $\mathrm{n}^{0} 3^{8 .}$

Cotryn B. G., Boucque Ch. V., I968. Variation de la composition du liquide du rumen en fonction dı moment de l'échantillonnage. Rev. A gr. Brussels, 21, nº II-1 2, I65I-I667.

Cottyn B. G., Boucque Ch. V., r971. Influence du broyage, de la finesse de mouture et de la mise en agglomérés sur la valeur alimentaire du foin de prairie. Rev. A gr. Brussels, 24, nº 8, 955-973.

Fenner H., Dickinson F. N., Barnes H. D., i967. Relationship of digestibility and certain rumen fluid components to level of feed intake and time of sampling after feeding. J. Dairy Sci., 50, 334-344.

Journet M., Demarquilly C., ig67. Valeur alimentaire des foins condensés. Influence du broyage et de la mise en agglomérés sur la digestion du foin de luzerne dans le rumen. Ann. Zootech., 16, 307-321.

Jarrige R., Demarquilly C., Journet M., Beranger G., 1973. 1 ${ }^{\text {st }}$ Intern. Green Crop Drying Congr., Oxford.

Journit M., Hobren A., I973. Utilisation des luzernes et des graminées déshydratées comme unique fourrage de la ration des vaches laitières et étude de leur digestion dans le rumen. Ann. Zootech., 22, 37-54.

Melcion J.-P., Delort-Laval J., 1972. Aliments complets agglomérés à base de paille et de céréales. Étude technologique. Ann. Zootech., 21, 535-542.

Minson 1). J., ig62. The effect of grinding, pelleting and wafering on the feeding value of roughage. Contr. $n^{\circ} \mathbf{8 4}$ Anim. Res. Institute Canada Dep. Agr. Ottawa, Ontario.

Moore L. A., r964. Symposium of forage utilization : General principles involved with ruminants and effect of feeding pelleted or wafered forage to dairy cattle. J. Anim. Sici., 23, $230-238$.

Thomson D. J., Beever D. E., Coelho da Silva J. F., Armstrong D. G., ig72. The effect in sheep of physical form on the sites of digestion of a dried lucerne diet. I. Sites of organic matter, energy and carbohydrate digestion. Irr. J. Nutr., 28, 3I-4I.

Tisserand J.-L., Zelter S. Z., 1965. Essai de normalisation d'une technique de mesures de la digestion des fourrages in vitro "rumen artificiel". Ann. Biol. anim. Bioch. Biophys., 5, ror-IIr.

Weston R. H., Hogan J. P., r967. The digestion of chopped and ground roughages by sheep. The movement of digesta through the stomach. Aust. J. Agr. Res., 18, 789-801.

Wright P. L., Pope A. L., Philipps P. H., r963. Effect of physical form of ration upon digestion and volatile fatty acid production in vivo and in vitro. J. Anim. Sci., 22, 586-59I. 